\title{
An FBN1 pseudoexon mutation in a patient with Marfan syndrome: confirmation of cryptic mutations leading to disease
}

\author{
Dong-chuan Guo $\cdot$ Prateek Gupta $\cdot$ Van Tran-Fadulu $\cdot$ Tera V. Guidry $\cdot$ \\ Magalie S. Leduc - Frederick V. Schaefer - Dianna M. Milewicz
}

Received: 5 June 2008/Accepted: 16 August 2008/Published online: 17 September 2008

(C) The Japan Society of Human Genetics and Springer 2008

\begin{abstract}
Marfan syndrome (MFS) results from heterozygous mutations in FBN1. However, genetic analyses of deoxyribonucleic acid (DNA) from approximately 10-30\% of MFS patients who meet diagnostic criteria do not reveal an identifiable $F B N 1$ mutation. In a patient who met the diagnostic criteria for MFS, bidirectional DNA sequencing of exons and intron-exon boundaries of $F B N 1$ failed to reveal a mutation. Assessment of the FBN1 message in dermal fibroblasts from the patient revealed insertion of a pseudoexon between exons 63 and 64. Sequencing of intron 63 identified a point mutation, IVS63+373, located near the middle of intron 63 of FBN1 that created a donor splice site in intron 63, leading to inclusion of a 93-bp fragment of intronic sequence in the $F B N 1$ message. Identification of a novel pseudoexon mutation in $F B N 1$, in association with a clinical diagnosis of MFS, confirms that cryptic mutations that are missed by the current DNAbased diagnostic methods have a causative role.
\end{abstract}

Keywords Cryptic mutation $\cdot F B N 1 \cdot$ Marfan syndrome · Pseudoexon

Dong-chuan Guo and Prateek Gupta contributed equally to this work.

D. Guo · P. Gupta · V. Tran-Fadulu · T. V. Guidry ·

M. S. Leduc · D. M. Milewicz ( $\square)$

The Department of Internal Medicine,

The University of Texas Medical School at Houston,

6431 Fannin, MSB 6.100, Houston, TX 77030, USA

e-mail: Dianna.M.Milewicz@uth.tmc.edu

F. V. Schaefer

Center for Genetic Testing at Saint Francis, Tulsa, OK, USA

\section{Introduction}

Marfan syndrome (MFS) is an autosomal dominant disorder with pleiotropic manifestations involving the cardiovascular, skeletal, and ocular systems (Murdoch et al. 1972; Pyeritz and McKusick 1979; Finkbohner et al. 1995). MFS results from mutations in $F B N 1$, which encodes fibrillin-1, a large glycoprotein found in the extracellular matrix (Dietz et al. 1991; Lee et al. 1991). Mutations in FBN1 that cause MFS are spread throughout the gene (http://www.umd. necker.fr). The majority of mutations are missense mutations that alter an amino acid in the calcium ion $\left(\mathrm{Ca}^{2+}\right)$ binding epidermal growth factor (EGF)-like domains (Hasham et al. 2002). Mutations that cause small in-frame deletions or insertions and mutations that lead to exon splicing errors have also been identified in FBN1 (Dietz et al. 1991; Guo et al. 2001).

More than $15 \%$ of inherited single-gene disorders in humans result from point mutations that disrupt proper splicing. However, mutations in introns that lead to insertion of an additional exon, termed pseudoexons, are rare. The first pseudoexon mutation leading to disease was identified in the ATM gene in an individual with familial ataxia telangiectasia (McConville et al. 1996). Since then, pseudoexon mutations have been identified in GHR (Metherell et al. 2001; David et al. 2007), DMD (TufferyGiraud et al. 2003; Eng et al. 2004; Coutinho et al. 2005), ATM (Eng et al. 2004; Coutinho et al. 2005), and PMM2 (Schollen et al. 2007), resulting in inherited growthhormone-insensitivity disease, Duchenne muscular dystrophy, ataxia telangiectasia, and congenital disorders of glycosylation type 1a and other genetic disorders (Harland et al. 2001; King et al. 2002).

In this report, deoxyribonucleic acid (DNA) analysis from a patient with MFS identified a point mutation 
located near the middle of intron 63 of $F B N 1$ that resulted in the insertion of a pseudoexon between exons 63 and 64 . This pseudoexon was predicted to lead to a premature termination codon at the beginning of the pseudoexon. Studies have identified FBN1 mutations in $91 \%$ of MFS patients, raising the possibility of cryptic FBN1 mutations not identified by current genomic sequencing approaches, or suggesting that mutations in other genes such as TGFBR2 may lead to classic MFS (Loeys et al. 2004). The data presented here confirm that cryptic FBN1 mutations, such as those that generate pseudoexons, can lead to MFS.

\section{Materials and methods}

Sample collection

The Institutional Review Board at the University of Texas Health Science Center, Houston, approved this study. After appropriate consent was obtained, peripheral blood specimens, skin biopsies, clinical information, and family history were obtained from the patients. Genomic DNA was extracted from blood samples according to the manufacturer's protocol using the PureGene genomic DNA isolation kit (Gentra Systems, Minneapolis, MN, USA).

\section{DNA sequencing and reverse transcription}

The 65 exons of FBN1 from the proband's and her mother's genomic DNA were polymerase chain reaction (PCR)-amplified and sequenced using intron-based, exonspecific primers (Nijbroek et al. 1995). PCR amplifications were carried out using HotStar Taq ${ }^{\mathrm{TM}}$ DNA polymerase (Qiagen Inc.Valencia, CA, USA). PCR products were sequenced by the ABI PRISM 3130xl Genetic Analyzer using the ABI PRISM BigDye Terminators v3.0 Cycle Sequencing Kit (Applied Biosystems). Dermal fibroblasts were explanted from skin biopsies. Total cellular ribonucleic acid (RNA) was purified by TRIzol Reagents (Life Technologies) and reverse-transcribed by Omniscript RT Kit (Qiagen) according to the manufacturer's protocol. The primers used for reverse transcription and reverse transcriptase (RT)-PCR were located at exon 63 (left primer 5'-TGCCTCAGCGCTCACATCTG) and exon 64 (right primer 5'-CTCGATATTGGAGGCATCAGT).

Quantification the transcription level of mutant mRNA

Pyrosequencing assay is a quantitative DNA sequencing technique applied for quantifying allele frequencies of single nucleotide polymorphisms (SNPs) [Guo et al. 2005]. We modified this method to quantify the transcriptional level of mutant messenger RNA (mRNA). To quantify transcriptional levels of total mRNA, an oligonucleotide, AGGGCGGTTACCTGTGTGGCTCTCCACCTGGT TAC TTCCGCATAGGCCAAGG, was synthesized that substituted a ' $\underline{\mathbf{C}}$ ' nucleotide for a $\mathrm{G}$ nucleotide normally found in FBN1 exon 63 sequence (Exon63C). This oligonucleotide served as a concentration standard to quantify the level of total FBN1 mRNA. A standard concentration of the Exon63C oligonucleotide was prepared at serial dilutions and then mixed with FBN1 complementary DNA (cDNA) from the proband's fibroblast. Pyrosequencing assays were performed to detect the peak height ratio of $\mathrm{C}$ and $\mathrm{G}$ alleles. The $\mathrm{C}$ peak represents the known copy number of the Exon63C standard and $\mathrm{G}$ peak the copy number of the proband's $F B N 1$ cDNA. The ratio of $\mathrm{C}$ to $\mathrm{G}$ alleles is equal to the ratio of the copy number of Exon63C standard to the proband's cDNA. Similarly, another oligonucleotide, CCAAGGTGATTCATCTCTCTCTAAAATACACCTC TCT GCCAACCCATGA, was synthesized that substituted a ' $\mathbf{C}$ ' nucleotide to replace a $G$ nucleotide in the pseudoexon sequence (Intron63C). This oligonucleotide served as standard to quantify the level of mutant FBN1 mRNA. Primers for the pyrosequencing assay of exon 63 were forward primer: biotin-GCGGTTACCTGTGTGG; reverse primer: CCTTGGCCTATGCGGAAGTA; and sequencing primer: TGCGGAAGTAACCAGGTG. Primers for the pyrosequencing assay of the pseudoexon were forward primer: biotin-CCAAGGTGATTCATCTC; reverse primer: TCATGGGTTGGCAGAGAGGT; and sequencing primer: TTGGCAGAGAGGTGTATT.

\section{Results}

Patient descriptions and family history

The proband was an 11-year-old Caucasian girl with aortic root dilatation $(3.2 \mathrm{~cm}$ at the sinuses of Valsalva with a body surface area of $1.32 \mathrm{~m}^{2}$ ) and mitral valve prolapse on echocardiogram. She had a stature greater than 95th percentile, scoliosis with a $22^{\circ}$ angle by X-ray, positive wrist and thumb sign, greater arm span to height ratio, pes planus with medial displacement, joint hypermobility, a narrow and slightly arched palate, and striae atrophicae. Based on these findings, the proband met the Ghent diagnostic criteria for MFS (De Paepe et al. 1996). The proband's mother was 42 years old with dolichostenomelia, narrow palate, myopia, hyperextensible metacarpal and interphalangeal joints, and mitral valve prolapse. The proband's maternal uncle underwent surgical repair of his ascending thoracic aorta; further medical information was not available. The maternal grandfather was $196 \mathrm{~cm}$ tall and died suddenly in his 60s. 
Identification and characterization of the FBN1 mutation

The 65 exons and adjacent intronic regions of $F B N 1$ were sequenced with the proband's DNA. No mutations were identified in the exons or intron-exon boundaries. To screen for a FBN1 splicing error, a nonquantitative RTPCR followed with DNA sequencing assays was performed on entire RNA isolated from fibroblasts explanted from the proband. When a cDNA fragment containing nucleotides 8,159-8,554 of the FBN1 message was analyzed, a fragment of the expected size was amplified, along with a larger DNA fragment (Fig. 1a). Sequencing of the larger DNA fragment revealed a 93-bp insertion between bp 8,379 and 8,380 , corresponding to the junction between exons 63 and 64. Basic local alignment search tool (BLAST) analysis (http://www.ncbi.nlm.nih.gov/BLAST/) identified that the inserted sequence was from intron 63 of FBN1 (Fig. 1b). The inserted intronic sequence was predicted to lead immediately to a premature termination codon.

To identify the disease-causing mutation in $F B N 1$, intron 63 was sequenced. $A \mathrm{G}$ to $\mathrm{T}$ transversion located $2 \mathrm{bp}$ downstream of $3^{\prime}$ terminus of the pseudoexon was identified
(Fig. 1c). This transversion was the only unreported nucleotide alteration found in intron 63. This transversion existed in both the proband and her mother but was not present in 200 unrelated Caucasian controls. The $\mathrm{G}$ to $\mathrm{T}$ transversion created an acceptor site for the pseudoexon (Fig. 1d). The transcription level of the mutant FBN1 allele was assayed by the pyrosequencing assay based semiquantitative RT-PCR method. In the proband's fibroblast, the transcriptional level of the mutant mRNA with the pseudoexon was $3 \%$ of the normal mRNA (data not shown).

\section{Discussion}

We report here the first pseudoexon mutation identified in $F B N 1$ leading to MFS. In addition, identification of this mutation provides the first evidence that "cryptic" FBN1 mutations undetected by diagnostic sequencing of genomic DNA can cause the disease. More than 600 mutations have been identified throughout the 65 exons of FBN1 in patients with MFS and related disorders, primarily through the 65 exons of $F B N 1$ and flanking intronic regions using genomic DNA (http://www.umd.necker.fr/disease.html). Approximately $11.5 \%$ of these mutations result in exon
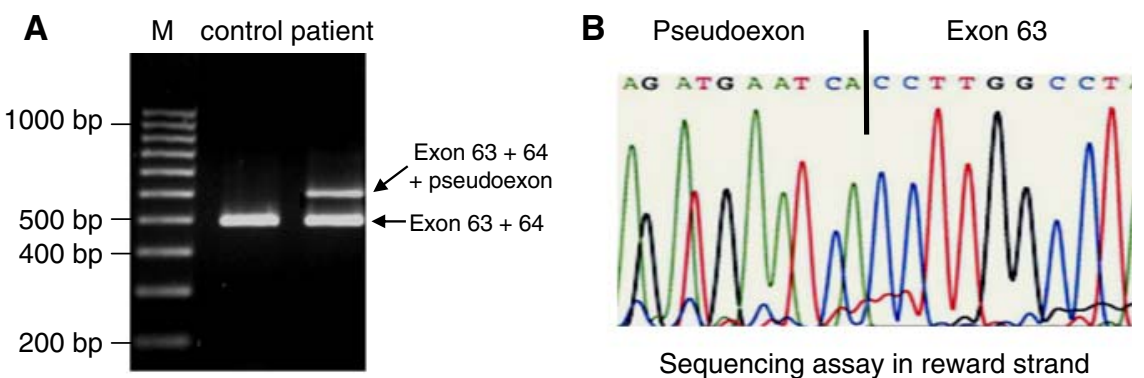

Sequencing assay in reward strand
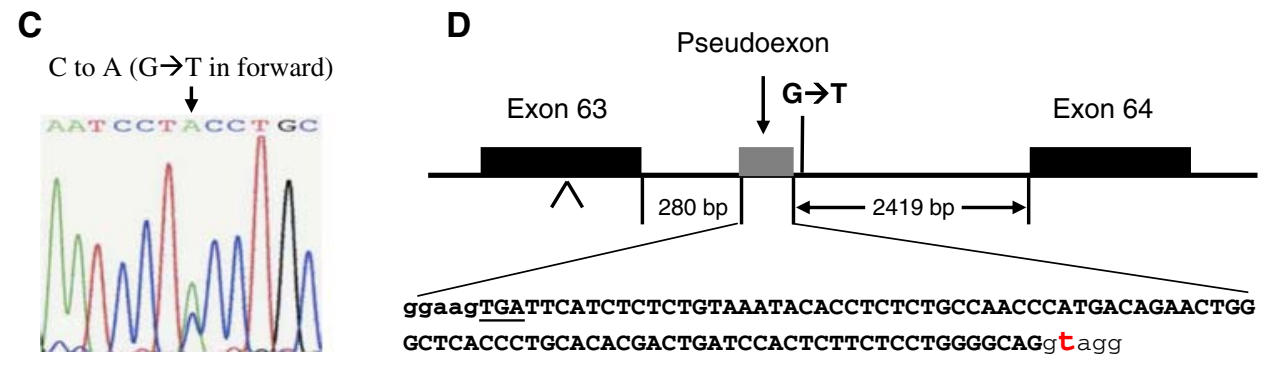

Fig. 1 A novel pseudoexon mutation is inserted between exon 63 and exon 64 of FBN1. a Reverse transcriptase polymerase chain reaction (RT-PCR) using primers located in exon 63 and 64 and ribonucleic acid (RNA) isolated from the patient's fibroblasts demonstrate a deoxyribonucleic acid (DNA) fragment of the expected size, plus a larger fragment that is not present in control RNA. $M$ is a 100 bp DNA ladder. b DNA sequencing assay of the larger complementary DNA (cDNA) fragment amplified from the patient's RNA confirmed a 93-bp pseudoexon insertion between exons 63 and 64. This sequencing assay was performed on a reverse cDNA strand. $\mathbf{c}$ DNA sequencing assay identified a $\mathrm{G} \rightarrow \mathrm{T}$ transversion in intron 63 of $F B N 1$. DNA sequencing assay was performed on the reverse strand. $\mathbf{d}$ The panel presents the location and sequence of the $\mathrm{G} \rightarrow \mathrm{T}$ transversion and pseudoexon in $F B N 1$. DNA sequence with capital letters is the pseudoexon. The red $t$ presents $\mathrm{G} \rightarrow \mathrm{T}$ transversion at the $3^{\prime}$ splice site. The first codon of the pseudoexon is a stop codon (underlined) 
splicing errors. A comprehensive molecular study on the FBN1 gene in a cohort of 93 MFS patients was initiated using single-strand conformation polymorphism and conformation-sensitive gel electrophoresis assays on 62 genomic DNAs, and 31 cDNAs derived from fibroblast RNA identified $F B N 1$ mutation in 73 patients fulfilling the Ghent diagnostic criteria. Direct sequencing of the exons and flanking introns in the remaining 20 patients was followed by Southern blot analysis for large deletions and insertions. This study identified FBN1 mutations in $91 \%$ of MFS patients (Loeys et al. 2004). A study of direct DNA sequencing of the exons and flanking introns of $F B N 1$, $F B N 2, T G F B R 1$, and TGFBR2 on 49 MFS or MFS-suspected patients identified $F B N 1$ mutations in $55 \%$ of patients and TGFBR1 and TGFBR2 mutations in $6 \%$ of patients [Sakai et al. 2006]. The MFS patients in these studies in whom no mutations were detected could have cryptic FBN1 mutations or locus heterogeneity for MFS (Boileau et al. 1993). Identification of a novel pseudoexon mutation in FBNI confirms a role of cryptic mutations leading to the condition. In addition, these results suggest that analysis of the FBN1 message should be considered when the standard genetic diagnosis fails to identify a mutation (Miller et al. 2007).

Only a few cases of pseudoexon mutations have been reported to cause human genetic disorders. The frequency of pseudoexon mutations may be underestimated because it is difficult to identify by direct DNA sequencing of exons and their flanking sequences of a gene. The mutations that cause pseudoexons can be located at intron-pseudoexon boundaries, within pseudoexon sequences that create a new cis element for RNA splicing, or in the pseudoexons through the deletion of intron-splicing processing element (Pagani et al. 2002). We analyzed the pseudoexon mutation and wild-type $F B N 1$ sequences using an ESE finder (http://rulai.cshl.edu/tools/ESE/) to investigate the potential mechanism of generating pseudoexon by the IVS63+373 alteration. A high-score motif, CACACGA, for exonic splicing enhancers SF2/ASF was found, which is located at the 61-66 bp of pseudoexon. The ESE finder score for this motif was 6.59 compared with the suggestive threshold score 1.96. This result suggests that the FBN1 IVS63+373 mutation creates an intronic GT sequence as a new acceptor site at the $5^{\prime}$ terminus and results in generation of a pseudoexon. Currently, defective genes have not been identified for many known loci that cause Mendelian inheritant diseases. In these cases, the intronic cryptic mutation is one of the potential defects that result in the disease.

Acknowledgments This work was support by RO1 HL62594 (D.M.M.), P50HL083794-01; Grant Number UL1 RR024148; UL1 RR024148 (CTSA) from the National Center for Research Resources
(NCRR) and NIH Roadmap for Medical Research and Doris Duke Foundation.

\section{References}

Boileau C, Jondeau G, Babron MC, Coulon M, Alexandre JA, Sakai L, Melki J, Delorme G, Dubourg O, Bonaiti-Pellie C (1993) Autosomal dominant Marfan-like connective-tissue disorder with aortic dilation and skeletal anomalies not linked to the fibrillin genes. Am J Hum Genet 53:46-54 See comments

Coutinho G, Xie J, Du L, Brusco A, Krainer AR, Gatti RA (2005) Functional significance of a deep intronic mutation in the ATM gene and evidence for an alternative exon 28a. Hum Mutat 25:118-124

David A, Camacho-Hubner C, Bhangoo A, Rose SJ, Miraki-Moud F, Akker SA, Butler GE, Ten S, Clayton PE, Clark AJ, Savage MO, Metherell LA (2007) An intronic growth hormone receptor mutation causing activation of a pseudoexon is associated with a broad spectrum of growth hormone insensitivity phenotypes. J Clin Endocrinol Metab 92:655-659

De Paepe A, Devereux RB, Dietz HC, Hennekam RC, Pyeritz RE (1996) Revised diagnostic criteria for the Marfan syndrome. Am J Med Genet 62:417-426

Dietz HC, Cutting GR, Pyeritz RE, Maslen CL, Sakai LY, Corson GM, Puffenberger EG, Hamosh A, Nanthakumar EJ, Curristin SM (1991) Marfan syndrome caused by a recurrent de novo missense mutation in the fibrillin gene. Nature 352: 337-339

Eng L, Coutinho G, Nahas S, Yeo G, Tanouye R, Babaei M, Dork T, Burge C, Gatti RA (2004) Nonclassical splicing mutations in the coding and noncoding regions of the ATM Gene: maximum entropy estimates of splice junction strengths. Hum Mutat 23:67-76

Finkbohner R, Johnston D, Crawford ES, Coselli J, Milewicz DM (1995) Marfan syndrome. Long-term survival and complications after aortic aneurysm repair. Circulation 91:728-733

Guo DC, Milewicz DM (2005) Pyrosequencing. In: Cohen A (ed) Encyclopedia of diagnostic genomics and proteomics. Marcel Dekker, New York, pp 1104-1107

Guo D, Tan FK, Cantu A, Plon SE, Milewicz DM (2001) FBN1 exon 2 splicing error in a patient with Marfan syndrome. Am J Med Genet 101:130-134

Harland M, Mistry S, Bishop DT, Bishop JA (2001) A deep intronic mutation in CDKN2A is associated with disease in a subset of melanoma pedigrees. Hum Mol Genet 10:2679-2686

Hasham SN, Guo DC, Milewicz DM (2002) Genetic basis of thoracic aortic aneurysms and dissections. Curr Opin Cardiol 17:677-683

King K, Flinter FA, Nihalani V, Green PM (2002) Unusual deep intronic mutations in the COL4A5 gene cause $\mathrm{X}$ linked Alport syndrome. Hum Genet 111:548-554

Lee B, Godfrey M, Vitale E, Hori H, Mattei MG, Sarfarazi M, Tsipouras P, Ramirez F, Hollister DW (1991) Linkage of Marfan syndrome and a phenotypically related disorder to two different fibrillin genes. Nature 352:330-334

Loeys B, De Backer J, Van Acker P, Wettinck K, Pals G, Nuytinck L, Coucke P, De Paepe A (2004) Comprehensive molecular screening of the FBN1 gene favors locus homogeneity of classical Marfan syndrome. Hum Mutat 24:140-146

McConville CM, Stankovic T, Byrd PJ, McGuire GM, Yao QY, Lennox GG, Taylor MR (1996) Mutations associated with variant phenotypes in ataxia-telangiectasia. Am J Hum Genet 59:320-330

Metherell LA, Akker SA, Munroe PB, Rose SJ, Caulfield M, Savage MO, Chew SL, Clark AJ (2001) Pseudoexon activation as a 
novel mechanism for disease resulting in atypical growthhormone insensitivity. Am J Hum Genet 69:641-646

Miller TE, You L, Myerburg RJ, Benke PJ, Bishopric NH (2007) Whole blood RNA offers a rapid, comprehensive approach to genetic diagnosis of cardiovascular diseases. Genet Med 9:23-33

Murdoch JL, Walker BA, McKusick VA (1972) Parental age effects on the occurrence of new mutations for the Marfan syndrome. Ann Hum Genet 35:331-336

Nijbroek G, Sood S, McIntosh I, Francomano CA, Bull E, Pereira L, Ramirez F, Pyeritz RE, Dietz HC (1995) Fifteen novel FBN1 mutations causing Marfan syndrome detected by heteroduplex analysis of genomic amplicons. Am J Hum Genet 57:8-21

Pagani F, Buratti E, Stuani C, Bendix R, Dork T, Baralle FE (2002) A new type of mutation causes a splicing defect in ATM. Nat Genet 30:426-429

Pyeritz RE, McKusick VA (1979) The Marfan syndrome: diagnosis and management. N Engl J Med 300:772-777
Sakai H, Visser R, Ikegawa S, Ito E, Numabe H, Watanabe Y, Mikami H, Kondoh T, Kitoh H, Sugiyama R, Okamoto N, Ogata T, Fodde R, Mizuno S, Takamura K, Egashira M, Sasaki N, Watanabe S, Nishimaki S, Takada F, Nagai T, Okada Y, Aoka Y, Yasuda K, Iwasa M, Kogaki S, Harada N, Mizuguchi T, Matsumoto N (2006) Comprehensive genetic analysis of relevant four genes in 49 patients with Marfan syndrome or Marfanrelated phenotypes. Am J Med Genet A 140:1719-1725

Schollen E, Keldermans L, Foulquier F, Briones P, Chabas A, Sanchez-Valverde F, Adamowicz M, Pronicka E, Wevers R, Matthijs G (2007) Characterization of two unusual truncating PMM2 mutations in two CDG-Ia patients. Mol Genet Metab 90:408-413

Tuffery-Giraud S, Saquet C, Chambert S, Claustres M (2003) Pseudoexon activation in the DMD gene as a novel mechanism for Becker muscular dystrophy. Hum Mutat 21:608-614 